\title{
Perspective: Water-Filtered Infrared-A-Radiation (wIRA) - Novel Treatment Options for Chlamydial Infections?
}

\author{
Jasmin Kuratli and Nicole Bore/* \\ Institute of Veterinary Pathology, University of Zurich, Zurich, Switzerland
}

Water-filtered infrared-A-radiation (wIRA) is a promising therapeutic method, which is particularly used as supportive treatment for wound closure, and wound infection treatment and prevention. High penetration properties of the heat field and beneficial effects on wound healing processes predispose wIRA irradiation to be a non-invasive treatment method for bacterial infections in superficial tissues. Since Chlamydia trachomatis still represents the leading cause of infectious blindness in third world countries (WHO http://www.who.int/topics/trachoma/en/) and wIRA displays beneficial effects on chlamydial infections in vitro without inducing cellular damage in ex vivo eye models and also shows beneficial effects on wound healing, this irradiation technique might represent a promising future treatment for trachoma patients. To this end, further studies investigating shorter irradiation times or irradiation of Chlamydia in chronic infections [the chlamydial stress response (Bavoil, 2014)] as well as safety studies in animal models should clearly be performed.

Keywords: Chlamydia trachomatis, wIRA irradiation, wIRA/VIS, wound healing, wound infection, alternative treatment strategies

\section{WATER-FILTERED INFRARED-A-RADIATION (wIRA)}

Electromagnetic radiation with wavelengths between $760 \mathrm{~nm}(0.760 \mu \mathrm{m})$ and $1000^{\prime} 000 \mathrm{~nm}$ $(1000 \mu \mathrm{m})$ is called infrared radiation (IR). This spectrum is divided into the three sub-ranges of IR-A $(760-1400 \mathrm{~nm})$, IR-B $(1400-3000 \mathrm{~nm})$, and IR-C (3000 - 1000'000 $\mathrm{nm}$ and $3.0-1000 \mu \mathrm{m})$ (Cobarg, 1995). The water-filtered IR-A-radiation (wIRA) shows a high degree of accordance with the terrestrial IR solar spectrum at noon as well as a strong reduction of emission within the water absorption bands at wavelengths of $840,970,1200$, and $1470 \mathrm{~nm}$ with negligible contributions of IR at wavelengths longer than $1400 \mathrm{~nm}$ (Cobarg, 1995). The radiation of a halogen bulb is filtered through a water-filled cuvette in special irradiators (Cobarg, 1995; Rzeznik, 1995). wIRA attains high tissue penetration with low thermal loads to the skin surface (in contrast to other sources of IR without the above-mentioned properties) and increases tissue temperature, oxygen partial pressure and tissue perfusion (Hellige et al., 1995; Hartel et al., 2007; Hoffmann, 2007). The most important clinical effects of wIRA reported in patients include reduction of pain, inflammation, hypersecretion and improved wound healing (Hartel et al., 2006, 2007; Hoffmann, 2007; Mercer et al., 2008). wIRA in the clinical setting of wound healing has been extensively reviewed elsewhere (Hartel et al., 2007; Hoffmann, 2007, 2009; von Felbert et al., 2007; Hoffmann et al., 2016). 


\section{APPLICATION OF WIRA IN PATIENTS}

A variety of clinical applications for wIRA has been described (reviewed in Hoffmann, 2007, 2017a), like improvement of wound healing in acute and chronic wounds (Hartel et al., 2007; von Felbert et al., 2007; Hoffmann, 2009; Daeschlein et al., 2012; Hoffmann et al., 2016), application in sports medicine and physiotherapy (Vaupel et al., 1995; Hoffmann, 2002), treatment of Morbus Bechterew (Falkenbach et al., 1996) or weight loss (Möckel et al., 2006), in the neonatology field (Singer et al., 2000; Löwe and Singer, 2008), for treatment of scleroderma lesions (Von Felbert et al., 2007, 2011), swallowing disorders and hypersalivation (Hoffmann, 2017b), therapy of recurrent breast cancer (Notter et al., 2017) and superficial tumors (Vaupel et al., 2018), or as a treatment method for actinic keratosis in combination with photodynamic therapy (PDT) (Von Felbert et al., 2010; Giehl et al., 2014). Moreover, wIRA can promote the penetration of topically applied substances (Otberg et al., 2008) and is also attractive for cosmetics and wellness applications (Piazena and Kelleher, 2010; Jung and Grune, 2012).

First introduced for chronic wound treatment in 1989, newer reports also recommend wIRA as a means to improve acute wound healing (Hartel et al., 2007; Daeschlein et al., 2012). After first anecdotal reports appeared describing single cases of better wound healing and reduced pain in patients following wIRA treatment, randomized control studies confirmed the beneficial effects of wIRA irradiation (reviewed in Hartel et al., 2007; von Felbert et al., 2007; Hoffmann, 2009; Hoffmann et al., 2016).

Re-irradiation of local recurrent breast cancer is often challenging and, in severely pre-treated patients, also limited due to risks of skin damage (Notter et al., 2017). A combination of superficial hyperthermia and radiation therapy allows reduction of the total radiation dose while achieving local control (Notter et al., 2017). wIRA irradiation has been assessed as a potential contactless heating technique, which is especially promising in the treatment of large superficial, neoplastic skin areas, and ulcerated or bleeding lesions due to additional wound healing promoting effects (Notter et al., 2017; Vaupel et al., 2018). First applications of water-filtered infrared-A and visible light irradiation (wIRA/VIS) in combination with PDT resulted in reduced local pain compared to PDT with light emitting diodes (LED) (Von Felbert et al., 2010) or with the light of a halogen bulb (Giehl et al., 2014). In addition, wIRA treatment improved PDT efficacy by inducing increased blood flow and tissue oxygenation (Von Felbert et al., 2010). wIRA can, moreover, be used in the treatment of other tumors such as malignant melanoma, vulvar carcinoma, skin metastases of different primary tumors, cutaneous T- and B-lymphomas, cutaneous large-area hemangiomatosis, inoperable squamous cell, basal cell and eccrine carcinoma (Vaupel et al., 2018).

Besides these myriad indications (reviewed by Hoffmann, 2007), wIRA application has been shown to be beneficial for the clinical treatment of donor site infections in split-thickness skin grafts (Aljasir et al., 2018). Hartel et al. (2006) postulated the tendency of reduced wound infection rates upon postoperative wIRA treatment. Preoperative wIRA application in a study with 400 patients undergoing abdominal surgery significantly reduced surgical site infections (Künzli et al., 2013). The combination of wIRA with PDT showed beneficial effects in the antimicrobial treatment of planktonic cultures and subgingival biofilms (Al-Ahmad et al., 2013, 2016; Karygianni et al., 2014). Furthermore, intracellular bacteria (Chlamydia pecorum and Chlamydia trachomatis) were significantly reduced in vitro by wIRA alone or in combination with visible light (wIRA/VIS) (Marti et al., 2014, 2015; Rahn et al., 2016; Kuratli et al., 2018).

\section{WIRA-INDUCED CLINICAL OBSERVATIONS}

The most important and reproducible clinical observations on wIRA treatment (increased tissue temperature, oxygen partial pressure, and tissue perfusion with decreased pain) are summarized in the first section of this report.

Wound centers are often relatively or absolutely hypothermic compared to the margins of the wound or unchanged skin areas (Hoffmann, 2007; Mercer et al., 2008), resulting in impaired wound healing; however, this can be treated by wIRAinduced elevation of local tissue temperature (Hoffmann, 2007). Reduced local oxygen partial pressures, often present in chronic wounds, inhibit sufficient energy production for wound healing processes (e.g., respiratory burst of granulocytes) and predispose to wound infections (Hoffmann, 2007). wIRA treatment can increase oxygen partial pressure, which has been demonstrated by implanted probe measurements (Hartel et al., 2006). The accumulation of lactate (leading to acidosis) and inflammatory metabolites as a result of impaired blood circulation can induce pain. Patients reported less pain perception during and after wIRA treatment (Hartel et al., 2006, 2007). Improved microcirculation due to wIRA treatment leads to removal of pain metabolites and long-lasting increase in tissue temperature through improved blood flow (Hoffmann, 2007).

\section{WORKING MECHANISM OF WIRA}

Physically, wIRA is a type of thermal radiation inducing thermal as well as non-thermal effects (Hoffmann, 2007). Thermal effects are explained by the increased tissue temperature and the increased kinetic energy (energy absorption) of water molecules upon heat radiation, leading to temperature-dependent changes in the affected tissue (Burri et al., 2004; Vaupel et al., 2018). Non-thermal effects are independent of temperature changes and a result of direct stimulation of cells or cellular structures (Burri et al., 2004). However, as stated by Jung et al. (2012), discriminating between thermal and non-thermal effects can be difficult when temperature changes are not strictly controlled. In cell culture, in contrast to skin models or even to a patient's skin, there is only one cell monolayer and the protective epidermis or temperature control by blood circulation is non-existent (Jung et al., 2010, 2012).

On the cellular level, cytochrome $\mathrm{c}$ oxidase has been extensively discussed as a potential target of visible light and 
near-infrared treatment (Karu, 1999, 2010; Karu et al., 2005). Cytochrome $c$ oxidase is a large multicomponent membrane protein and the terminal enzyme of the respiratory chain in eukaryotic cells which mediates the transfer of electrons from cytochrome $\mathrm{c}$ to molecular oxygen (Karu, 1999). Multiple studies hypothesize that intermediate forms of cytochrome c (not fully oxidized, not fully reduced) are responsible for photo-acceptor properties (Karu, 1999, 2010; Karu et al., 2005) and cytochrome c oxidase has recently been confirmed as a photo-acceptor (Passarella and Karu, 2014). Nevertheless, four potential primary light action mechanisms are discussed, which are likely to occur as combinations: (1) alteration of redox properties and acceleration of electrons, (2) changed biochemical activity by local transient heating of chromophores (through conversion of excitation energy into heat energy), (3) production of reactive oxygen species (mainly $\mathrm{O}_{2}{ }^{-}$) and subsequent $\mathrm{H}_{2} \mathrm{O}_{2}$ production, and (4) photodynamic action and singlet oxygen production (Karu, 1999).

Biological responses of cells after irradiation might play a role as secondary mechanisms (Karu, 1999). The transduction of light action from mitochondria to the nucleus leading to DNA synthesis needs to be elucidated (Karu, 1999). A possible explanation is based on the fact that redox chains (including respiratory chain) are capable of controlling cellular homeostasis. Changes in the redox potential in mitochondria also affect the redox state in the cytoplasm and induce the signal transduction to the nucleus (Karu, 1999). Furthermore, the physiological significance of photosensitivity in enzymes of the respiratory chain is an important question to assess (Karu et al., 2005). Menezes et al. (1998) suggested that IR, for example during sunrise, is a natural process protecting cells from subsequent UV-radiation throughout the day, which is in accordance with conclusions by Applegate et al. (2000) and Burri et al. (2004). Multiple studies have confirmed that IR protects cells from UV cytotoxicity (Danno et al., 1992; Menezes et al., 1998; Frank et al., 2004, 2006). Wavelength-specific influence on cytochrome c oxidase has been reported by Karu et al. (2001; 2003; 2004; 2005) in multiple studies (Karu, 1999, 2008; Passarella and Karu, 2014). Heselich et al. (2012) demonstrated increased genomic instability, increased ROS formation, higher amounts of X-irradiated cells entering the S-phase, and impaired DNA double-strand break repair upon near-infrared-irradiation followed by clinically relevant X-ray doses. These synergistic effects are likely to affect the results of radiotherapy in cancer treatment (Heselich et al., 2012), which is further confirmed by the abovementioned combination of radiotherapy and heat-therapy with irradiation using wIRA/VIS irradiators (Notter et al., 2017; Vaupel et al., 2018).

Multiple authors have reported increased concentrations of reactive oxygen species (ROS) or decreased concentrations of antioxidative substances upon IR in human skin models in vitro or in vivo (Schroeder et al., 2007, 2008; Darvin et al., 2009, 2011) and have suspected harmful effects of IR and wIRA on cell layers. Other authors investigated the secretion of matrix-metalloproteinases (MMP) (reviewed in Piazena and Kelleher, 2010). An in vivo study investigating IR radiation on albino guinea pigs revealed harmful effects of IR similar to UV-damage (Kligman, 1982). Piazena and Kelleher (2010) give an overview of literature concerning the safety aspects of water-filtered infrared-A or other sources of IR, which the reader is referred to (Piazena and Kelleher, 2010). Most of the studies performed are considered unsuitable as models for infrared or water-filtered IR on the human skin, since most of them lack important information about dose, exposure-time, spectrum, and application of irradiation. Corresponding controls need to be characterized and would help to distinguish the secondary effects of VIS or UV radiation from IR/wIRA effects (Piazena and Kelleher, 2010). Other authors investigating ROS formation in fibroblasts were able to demonstrate increased ROS concentrations upon temperature increase in the samples alone, which leads to the conclusion of ROS activation being an unspecific thermal effect of the radiation (Jung et al., 2010). Therefore, the importance of suitable temperature control needs to be particularly emphasized and is vital in the interpretation of results for in vitro and in vivo studies (Piazena and Kelleher, 2010; Jung and Grune, 2012).

Even though in vitro studies using high doses of wIRA or wIRA/VIS did not induce cell death (Rahn et al., 2016), discomfort in patient due to heating of irradiated tissues might be possible. This can be prevented by either using lower doses [e.g., $1500-2000 \mathrm{~W} / \mathrm{m} 2$ as in Notter et al. (2017)], by keeping a sufficient distance to the irradiator [see Hartel et al. (2006); Künzli et al. (2013) - Table 1, Aljasir et al. (2018)] or by limiting the irradiation duration.

\section{WIRA AS AN ANTIBACTERIAL TREATMENT METHOD}

A summary of studies using wIRA as an antibacterial treatment method is presented in Table 1. Al-Ahmad et al. (2013) were the first authors to investigate the efficacy of visible light in combination with water-filtered infrared-A (VIS and wIRA) in the killing of bacteria. In their study, antimicrobial photodynamic therapy (aPDT) was tested using toluidine blue (TB) at different concentrations as a photosensitizer and visible light and waterfiltered infrared-A radiation as light sources. Bacterial samples tested were planktonic cultures of Streptococcus mutans and Enterococcus faecalis, bacteria occurring in human saliva or originating from bovine enamel slabs. These investigations were carried out in the oral cavity of healthy volunteers to investigate the effects of aPDT on bacterial colonization in situ. All the aforementioned bacteria were severely diminished in load after aPDT treatment, with a maximal reduction of $3.7-5 \log _{10}$ in salivary bacteria (Al-Ahmad et al., 2013). A further study also investigated the action of aPDT consisting of chlorine e6 and wIRA/VIS on multiple periodontal pathogens (Aggregatibacter actinomycetemcomitans, Porphyromonas gingivalis, Eikenella corrodens, Actinomyces odontolyticus, Fusobacterium nucleatum, Parvimonas micra, Slackia exigua, and Atopobium rimae) and subgingival biofilms in situ, which are protected against antibiotic treatment or disinfection by various mechanisms (Al-Ahmad et al., 2016). Karygianni et al. (2014) investigated the effect of aPDT (using toluidine blue or chlorine e6) with wIRA/VIS 


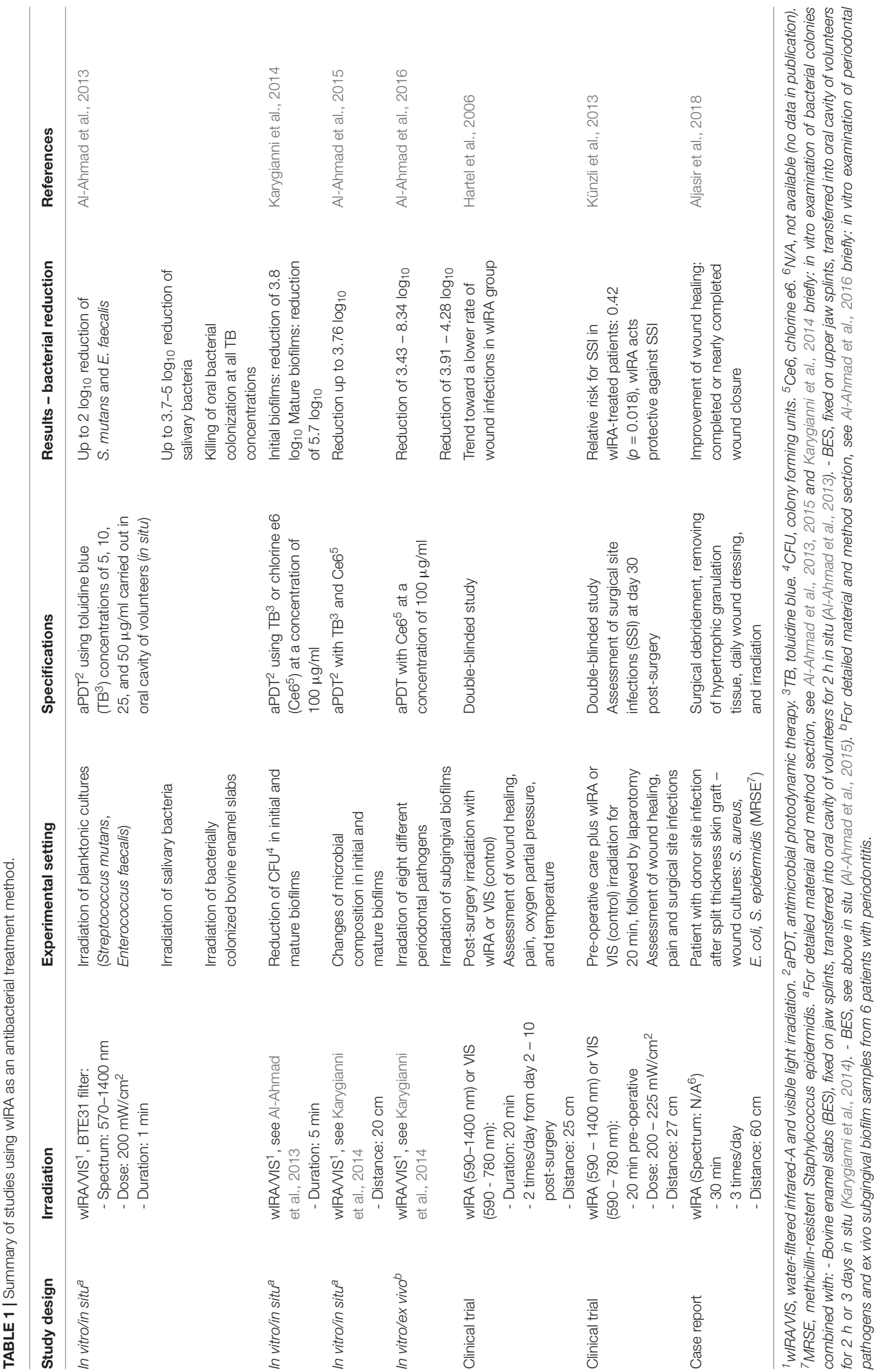




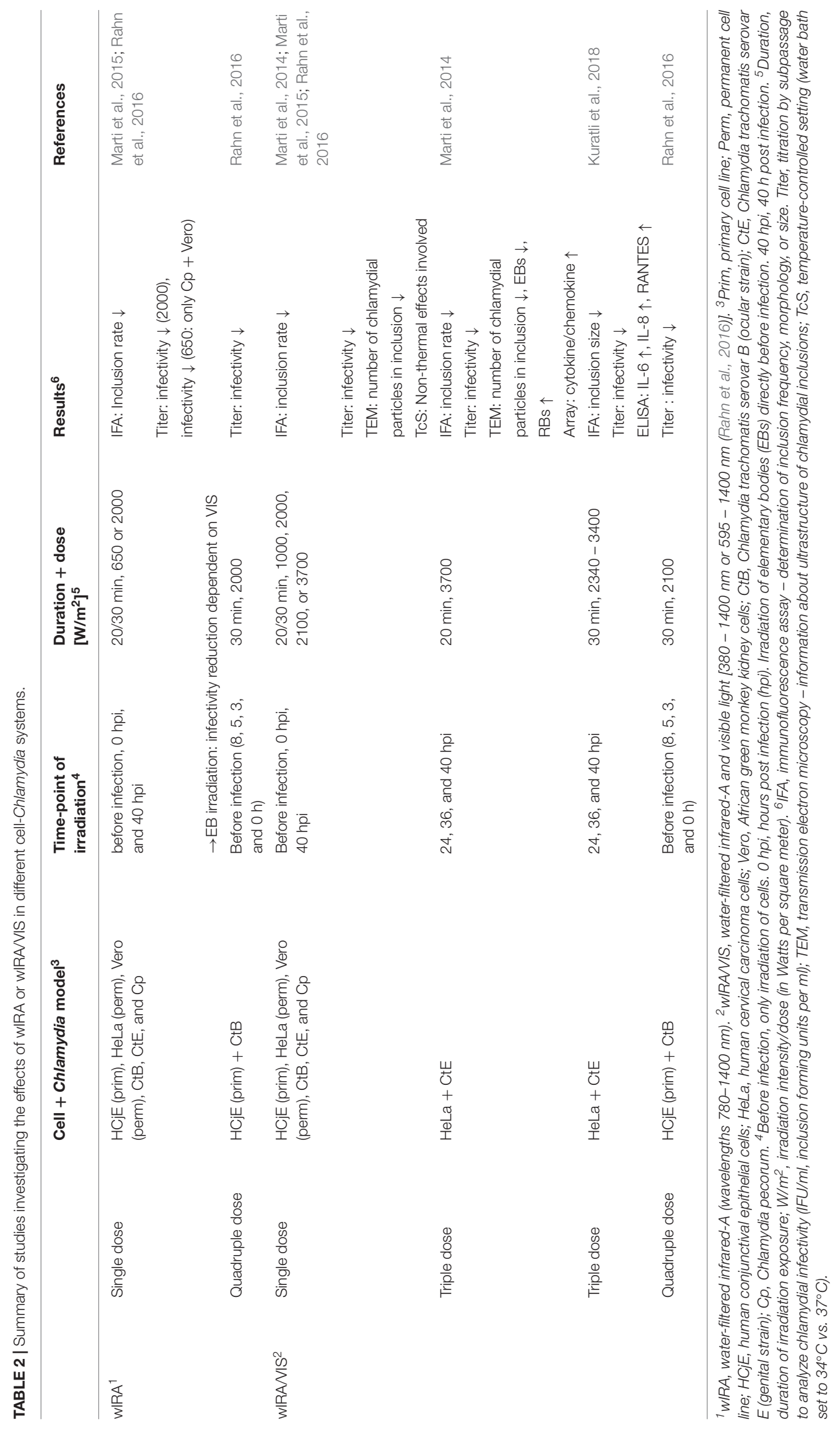




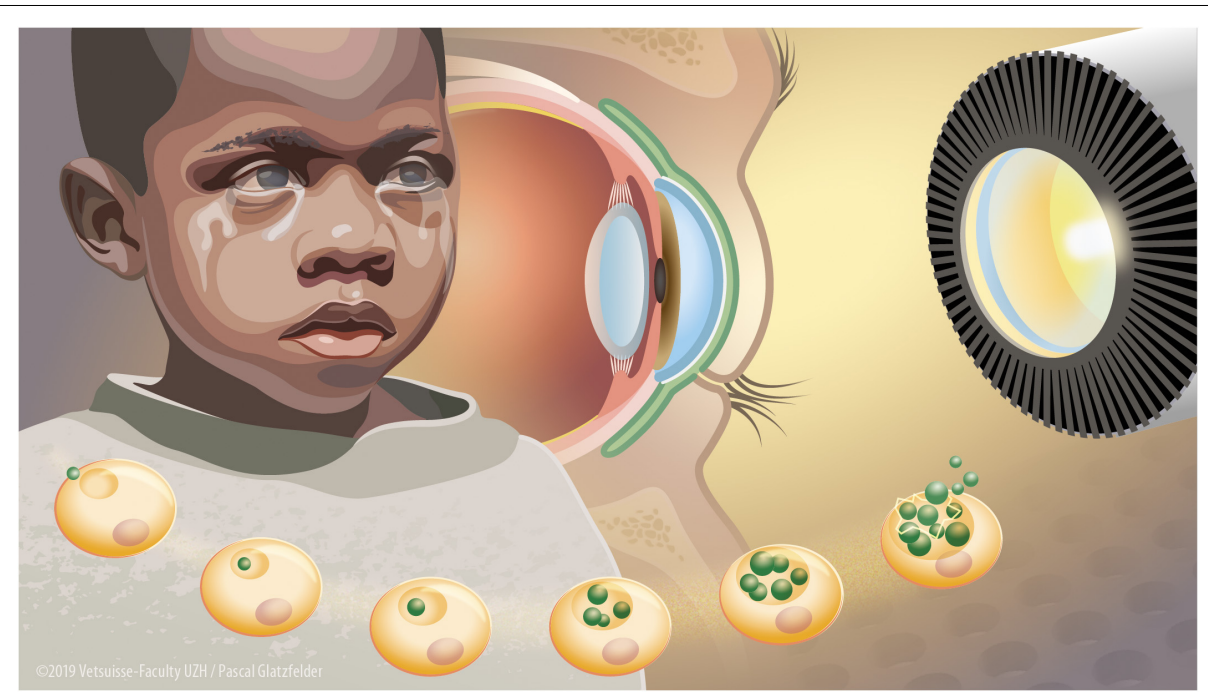

FIGURE 1 | Water-filtered infrared-A and visible light irradiation (WIRANIS) as a potential future treatment device to combat blinding trachoma. Blinding trachoma is a neglected tropical disease present in Sub-Saharan Africa, in particular affecting children. It is caused by recurrent infections of the conjunctival epithelium by C. trachomatis leading to fibrosis and scarring of the conjunctiva, ocular discharge, trichiasis, and corneal opacity with blindness (left aspect of the figure). C. trachomatis is an obligate intracellular bacterium with a complex life cycle including the attachment of an elementary body (EB) to an epithelial cell, forming an intracytoplasmic inclusion with differentiation into reticulate bodies and multiplication by binary fission and finally lysis of the inclusion with the release of EBs (bottom aspect of the figure). wIRAVIS irradiation produced by radiation of a halogen bulb, filtered through a water-filled cuvette in special irradiators (right aspect of the figure) could reduce chlamydial burden in vitro by affecting mature inclusions and as well as by reducing infectious EB load. Future in vivo wIRAVIS treatment of trachoma patients would be applied by irradiation of the $C$. trachomatis-infected conjunctiva (in green) through a closed eyelid reaching the infected part (inner conjunctival lining) without harming the inner structures of the eye such as the cornea, the vitreous body or the retina (central aspect of the figure).

on initial and mature oral biofilms and observed bacterial reductions of 3.8 and $5.7 \log _{10}$, respectively. In situ oral biofilms were severely reduced and the proportion of surviving bacteria was altered compared to controls upon wIRA/VIS radiation, which further encourages the clinical use of wIRA/VIS as treatment for periimplantitis, and periodontitis (Al-Ahmad et al., 2015). Common light sources in aPDT include lightemitting diodes (LED) and wide-band halogen lamps (AlAhmad et al., 2016). wIRA/VIS was proven to be beneficial for aPDT therapy in multiple studies (Al-Ahmad et al., 2013, 2016; Karygianni et al., 2014), also including the advantages of using a broader wavelength-spectrum than LED, reduced risk of tissue overheating compared to halogen lamps, and its well-known beneficial effects on wound healing (Al-Ahmad et al., 2016).

In addition to the above-mentioned beneficial aspects of wIRA/VIS in aPDT, the application of wIRA/VIS alone (without photosensitizer) had beneficial effects on wound infections after abdominal surgery (Hartel et al., 2006; Künzli et al., 2013). The case report of a patient with a donor site infection in a split-thickness skin graft is especially worth mentioning, since Staphylococcus aureus, Escherichia coli, and Staphylococcus epidermidis (Methicillin-resistant, MRSE) were isolated from the wound site and the combination of surgical intervention, daily wound dressing, and combined wIRA/VIS treatment led to almost complete healing of the infected sites within 5 weeks (Aljasir et al., 2018).

Finally, not only extracellular bacteria, wound infections and biofilm formation can be treated with wIRA/VIS, but this irradiation technique has also been demonstrated to reduce acute chlamydial infections in vitro (Marti et al., 2014, 2015; Rahn et al., 2016; Kuratli et al., 2018). The reducing effects of wIRA/VIS on chlamydial infectivity were confirmed in multiple in vitro studies, using animal (Vero cells and C. pecorum) and human cell culture models (a genital model with HeLa cells and C. trachomatis Serovar E or an ocular model with human conjunctival epithelial cells and C. trachomatis Serovar B), different chlamydial infectious doses, different irradiation time points, irradiation doses or wavelengths (wIRA alone (black filter, 780-1400 nm), wIRA/VIS (orange filter, 595-1400 nm or no filter, $380-1400 \mathrm{~nm}$ ), and temperature controlled settings (Marti et al., 2014, 2015; Rahn et al., 2016; Kuratli et al., 2018). Results of previous studies investigating wIRA or wIRA/VIS for the treatment of chlamydial infections are summarized in Table 2.

Since chlamydiae are obligate intracellular bacteria, the first investigations of wIRA treatment for chlamydial infections focused on possible deteriorating effects of wIRA irradiation on host cells. Detrimental effects on host cells were not present in HeLa (human cervical carcinoma cells) and Vero (African green monkey kidney) cells (Marti et al., 2014). In primary human conjunctival epithelial cells $(\mathrm{HCjE})$, wIRA treatment reduced cell metabolism as measured by Alamarblue assay, whereas wIRA/VIS did not. However, the additional counting of cell numbers per high power field $(400 \times$ magnification) did not show decreased cell numbers after either wIRA/VIS or wIRA irradiation treatment (Rahn et al., 2016). Following these findings, further investigations included ex vivo models, which are important for potential future application of wIRA as a trachoma treatment method (Figure 1). Although a temperature 
increase was demonstrated in constantly perfused pig eyes upon high irradiation doses, no wIRA/VIS-dependent phosphorylation of stress kinases was observed in retinal explants from adult or postnatal (10-day-old) mice (Rahn et al., 2016).

Irradiation of $\mathrm{HCjE}$ cells alone prior to chlamydial infection reduced subsequent chlamydial infectivity, indicating a cellassociated, protective effect of wIRA/VIS against chlamydial infections (Rahn et al., 2016). Irradiating elementary bodies (EBs - infective particles in the chlamydial life cycle) prior to infection of host cells also reduced chlamydial infectivity (Marti et al., 2014, 2015; Rahn et al., 2016) and the combination of both prior to infection further increased this effect (Rahn et al., 2016).

The irradiation of mature chlamydial inclusions in vitro with either wIRA or wIRA/VIS at doses of 2000 or $3700 \mathrm{~W} / \mathrm{m}^{2}$ led to a decrease of the number of infective particles in the chlamydial inclusions without causing changes in the distribution of chlamydial maturation stages (Marti et al., 2014, 2015). Triple dose irradiation of growing Chlamydia, on the other hand, led to increased proportions of reticulate bodies (RBs - the reproducing form of Chlamydia) and decreased EB formation, whereas no increased formation of aberrant bodies $[A B$ - a sign of the chlamydial stress response (Bavoil, 2014)] could be observed (Marti et al., 2014). The distribution differences in chlamydial development stages indicate a yet unknown effect of wIRA/VIS on the chlamydial development cycle (Marti et al., 2014).

\section{FUTURE RESEARCH DIRECTIONS}

To elucidate the mechanism of wIRA/VIS in antibacterial effects or in wound healing, transcriptomics or metabolomics would provide useful information regarding gene expression and protein translation upon irradiation. Since cytochrome $c$ oxidase has been claimed to be the primary photo-acceptor molecule, investigating the molecule itself, and the broader mitochondrial metabolism might provide useful information regarding biological effects following wIRA irradiation.

\section{REFERENCES}

Al-Ahmad, A., Bucher, M., Anderson, A. C., Tennert, C., Hellwig, E., Wittmer, A., et al. (2015). Antimicrobial photoinactivation using visible light plus waterfiltered infrared-A (VIS + wIRA) alters in situ oral biofilms. PLoS One 10:e0132107. doi: 10.1371/journal.pone.0132107

Al-Ahmad, A., Tennert, C., Karygianni, L., Wrbas, K. T., Hellwig, E., and Altenburger, M. J. (2013). Antimicrobial photodynamic therapy using visible light plus water-filtered infrared-A (wIRA). J. Med. Microbiol. 62, 467-473. doi: 10.1099/jmm.0.048843-0

Al-Ahmad, A., Walankiewicz, A., Hellwig, E., Follo, M., Tennert, C., Wittmer, A., et al. (2016). Photoinactivation using visible light plus water-filtered infrared-A (vis+wira) and chlorine e6 (Ce6) eradicates planktonic periodontal pathogens and subgingival biofilms. Front. Microbiol. 7:1900. doi: 10.3389/fmicb.2016. 01900

Aljasir, A., Pierson, T., Hoffmann, G., and Menke, H. (2018). Management of donor site infections in split-thickness skin graft with water-filtered infrared-A (wIRA). GMS Ger. Med. Sci. 7, doi: 10.3205/iprs000123

Applegate, L. A., Scaletta, C., Panizzon, R., Frenk, E., Hohlfeld, P., and Schwarzkopf, S. (2000). Induction of putative protective protein ferritin by infrared radiation: implications in skin repair. Int. J. Mol. Med. 5, 247-251.
Various bacterial species have been demonstrated to display thermo-sensitivity, therefore local thermal therapies (as e.g., wIRA therapy) are important fields for future investigations (Gazel and Yilmaz, 2018). Heat treatment has further been proven beneficial for Leishmania-induced skin lesions (Aronson et al., 2010) and buruli ulcer, another tropical disease, which is caused by Mycobacterium ulcerans (Junghanss et al., 2009; Vogel et al., 2016). These neglected tropical diseases might represent further possible indications for the use of wIRA.

In view of current worldwide antibiotic resistance problems, studies investigating wound infection treatment with heat and light have become more important in the last few decades, with promising results for these treatments. Further investigations are needed to gain deeper insight into the working mechanisms of such methods, their safety aspects, and applicability in patients.

\section{AUTHOR CONTRIBUTIONS}

JK and NB drafted the manuscript.

\section{FUNDING}

Funding was provided by the Dr. med. h. c. Erwin Braun Foundation, Basel, Switzerland.

\section{ACKNOWLEDGMENTS}

We thank Werner Müller and Dr. rer. nat. Eduard Wolf for supporting this work with their broad knowledge in the field of infrared radiation as well as the Dr. med. h. c. Erwin Braun Foundation, Basel, Switzerland for providing funding for this project. We also thank Pascal Glatzfelder for designing the graphical abstract.

Aronson, N. E., Wortmann, G. W., Byrne, W. R., Howard, R. S., Bernstein, W. B., Marovich, M. A., et al. (2010). A randomized controlled trial of local heat therapy versus intravenous sodium stibogluconate for the treatment of cutaneous Leishmania major infection. PLoS Negl. Trop. Dis. 4:e628. doi: 10. 1371/journal.pntd.0000628

Bavoil, P. M. (2014). What's in a word: the use, misuse, and abuse of the word \&quot;persistence\& quot; in chlamydia biology. Front. Cell. Infect. Microbiol. 4:27. doi: 10.3389/fcimb.2014.00027

Burri, N., Gebbers, N., and Applegate, L. A. (2004). Chronic infrared-A radiation repair: implications in cellular senescence and extracellular matrix. Recent Res. Dev. Photochem. Photobiol. 7, 219-231.

Cobarg, C. C. (1995). "Physikalische Grundlagen der wassergefilterten InfrarotA-Strahlung," in Wärmetherapie Mit Wassergefilterter Infrarot-A-Strahlung. Grundlagen und Anwendungsmöglichkeiten, eds P. Vaupel and W. Krüger (Stuttgart: Hippokrates Verlag), 19-28.

Daeschlein, G., Alborova, J., Patzelt, A., Kramer, A., and Lademann, J. (2012). Kinetics of physiological skin flora in a suction blister wound model on healthy subjects after treatment with water-filtered infrared-a radiation. Skin Pharmacol. Physiol. 25, 73-77. doi: 10.1159/000332753

Danno, K., Horio, T., and Imamura, S. (1992). Infrared radiation suppresses ultraviolet B-induced sunburn-cell formation. Arch. Dermatol. Res. 284, 92-94. 
Darvin, M. E., Fluhr, J. W., Meinke, M. C., Zastrow, L., Sterry, W., and Lademann, J. (2011). Topical beta-carotene protects against infra-red-light-induced free radicals. Exp. Dermatol. 20, 125-129. doi: 10.1111/j.1600-0625.2010.01191.x

Darvin, M. E., Patzelt, A., Meinke, M., Sterry, W., and Lademann, J. (2009). Influence of two different IR radiators on the antioxidative potential of the human skin. Laser Phys. Lett. 6, 229-234. doi: 10.1002/lapl.200810124

Falkenbach, A., Dorigoni, H., Werny, F., and Gütl, S. (1996). Wassergefilterte infrarot-a-bestrahlung bei morbus bechterew und degenerativen wirbelsäulenveränderungen: effekte auf beweglichkeit und druckschmerzhaftigkeit. ÖZPMR 6, 96-102.

Frank, S., Menezes, S., Lebreton-De Coster, C., Oster, M., Dubertret, L., and Coulomb, B. (2006). Infrared radiation induces the p53 signaling pathway: role in infrared prevention of ultraviolet B toxicity. Exp. Dermatol. 15, 130-137. doi: 10.1111/j.1600-0625.2005.00397.x

Frank, S., Oliver, L., Lebreton-De Coster, C., Moreau, C., Lecabellec, M. T., Michel, L., et al. (2004). Infrared radiation affects the mitochondrial pathway of apoptosis in human fibroblasts. J. Invest. Dermatol. 123, 823-831. doi: 10. 1111/j.0022-202X.2004.23472.x

Gazel, D., and Yllmaz, M. (2018). Are infectious diseases and microbiology new fields for thermal therapy research? Int. J. Hyperth. 34, 918-924. doi: 10.1080/ 02656736.2018.1440015

Giehl, K. A., Kriz, M., Grahovac, M., Ruzicka, T., and Berking, C. (2014). A controlled trial of photodynamic therapy of actinic keratosis comparing different red light sources. Eur. J. Dermatol. 24, 335-341.

Hartel, M., Hoffmann, G., Wente, M. N., Martignoni, M. E., Büchler, M. W., and Friess, H. (2006). Randomized clinical trial of the influence of local waterfiltered infrared A irradiation on wound healing after abdominal surgery. $\mathrm{Br}$. J. Surg. 93, 952-960. doi: 10.1002/bjs.5429

Hartel, M., Illing, P., Mercer, J. B., Lademann, J., Daeschlein, G., and Hoffmann, G. (2007). Therapy of acute wounds with water-filtered infrared-A (wIRA). GMS Krankenhhyg. Interdiszip. 2:Doc53.

Hellige, G., Becker, G., and Hahn, G. (1995). "Temperaturverteilung und Eindringtiefe wassergefilterter Infrarot-A-Strahlung," in Wärmetherapie mit Wassergefilterter Infrarot-A-Strahlung. Grundlagen und Anwendungsmöglichkeiten, eds P. Vaupel and W. Krüger (Stuttgart: Hippokrates Verlag).

Heselich, A., Frohns, F., Frohns, A., Naumann, S. C., and Layer, P. G. (2012). Nearinfrared exposure changes cellular responses to ionizing radiation. Photochem. Photobiol. 88, 135-146. doi: 10.1111/j.1751-1097.2011.01031.x

Hoffmann, G. (2002). Improvement of regeneration by local hyperthermia induced by waterfiltered infrared $\mathrm{A}$ (wIRA). Int. J. Sports Med. 23(Suppl. 2):S145.

Hoffmann, G. (2007). Principles and working mechanisms of water-filtered infrared-A (wIRA) in relation to wound healing. GMS Krankenhhyg. Interdiszip. 2:Doc54.

Hoffmann, G. (2009). Water-filtered infrared-A (wIRA) in acute and chronic wounds. GMS Krankenhhyg. Interdiszip. 4:Doc12. doi: 10.3205/dgkh00 0137

Hoffmann, G. (2017a). Clinical applications of water-filtered infrared-A (wIRA) - a review. Phys. Medizin Rehabil. Kurortmedizin 27, 265-274. doi: 10.1055/s-0043113047

Hoffmann, G. (2017b). Water-filtered infrared-A (wIRA) overcomes swallowing disorders and hypersalivation - a case report. Ger. Med. Sci. 15:Doc11. doi: $10.3205 / 000252$

Hoffmann, G., Hartel, M., and Mercer, J. B. (2016). Heat for wounds-waterfiltered infrared-A (wIRA) for wound healing-a review. Ger. Med. Sci. 14:Doc08. doi: $10.3205 / 000235$

Jung, T., and Grune, T. (2012). Experimental basis for discriminating between thermal and athermal effects of water-filtered infrared A irradiation. Ann. N. Y. Acad. Sci. 1259, 33-38. doi: 10.1111/j.1749-6632.2012.06581.x

Jung, T., Höhn, A., Lau, A. M., Piazena, H., and Grune, T. (2012). An experimental setup for the measurement of nonthermal effects during water-filtered infrared a-irradiation of mammalian cell cultures. Photochem. Photobiol. 88, 371-380. doi: 10.1111/j.1751-1097.2011.01072.x

Jung, T., Höhn, A., Piazena, H., and Grune, T. (2010). Effects of water-filtered infrared A irradiation on human fibroblasts. Free Radic. Biol. Med. 48, 153-160. doi: 10.1016/j.freeradbiomed.2009.10.036
Junghanss, T., Boock, A. U., Vogel, M., Schuette, D., Weinlaeder, H., and Pluschke, G. (2009). Phase change material for thermotherapy of buruli ulcer: a prospective observational single centre proof-of-principle trial. PLoS Negl. Trop. Dis. 3:e380. doi: 10.1371/journal.pntd.0000380

Karu, T. (1999). Primary and secondary mechanisms of action of visible to near-IR radiation on cells. J. Photochem. Photobiol. B Biol. 49, 1-17. doi: 10.1016/S10111344(98)00219-X

Karu, T. I. (2008). Mitochondrial signaling in mammalian cells activated by red and near-IR radiation. Photochem. Photobiol. 84, 1091-1099. doi: 10.1111/j.17511097.2008.00394.x

Karu, T. I. (2010). Multiple roles of cytochrome c oxidase in mammalian cells under action of red and IR-A radiation. IUBMB Life 62, 607-610. doi: 10.1002/ iub.359

Karu, T. I., Pyatibrat, L. V., and Kalendo, G. S. (2001). Cell attachment to extracellular matrices is modulated by pulsed radiation at $820 \mathrm{~nm}$ and chemicals that modify the activity of enzymes in the plasma membrane. Lasers Surg. Med. 29, 274-281. doi: 10.1002/lsm.1119

Karu, T. I., Pyatibrat, L. V., Kolyakov, S. F., and Afanasyeva, N. I. (2005). Absorption measurements of a cell monolayer relevant to phototherapy: reduction of cytochrome c oxidase under near IR radiation. J. Photochem. Photobiol. B Biol. 81, 98-106. doi: 10.1016/j.jphotobiol.2005.07.002

Karu, T. I., Pyatibrat, L. V., and Ryabykh, T. P. (2003). Melatonin modulates the action of near infrared radiation on cell adhesion. J. Pineal Res. 34, 167-172. doi: 10.1034/j.1600-079X.2003.00023.x

Karu, T. L., Pyatibrat', L. V., and Afanasyeva, N. L. (2004). A novel mitochondria1 signaling pathway activated by visi ble-to-near infrared radiation. Photochem. Photobiol. 80, 366-372.

Karygianni, L., Ruf, S., Follo, M., Hellwig, E., Bucher, M., Anderson, A. C., et al. (2014). Novel broad-spectrum antimicrobial photoinactivation of in situ oral biofilms by visible light plus water-filtered infrared A. Appl. Environ. Microbiol. 80, 7324-7336. doi: 10.1128/AEM.02490-14

Kligman, L. H. (1982). Intensification of ultraviolet-induced dermal damage by infrared radiation. Arch. Dermatol. Res. 272, 229-238. doi: 10.1007/ BF00509050

Künzli, B. M., Liebl, F., Nuhn, P., Schuster, T., Friess, H., and Hartel, M. (2013). Impact of preoperative local water-filtered infrared a irradiation on postoperative wound healing: a randomized patient-and observerblinded controlled clinical trial. Ann. Surg. 258, 887-894. doi: 10.1097/SLA. 0000000000000235

Kuratli, J., Pesch, T., Marti, H., Leonard, C. A., Blenn, C., Torgerson, P., et al. (2018). Water filtered infrared a and visible light (wIRA/VIS) irradiation reduces chlamydia trachomatis infectivity independent of targeted cytokine inhibition. Front. Microbiol. 9:2757. doi: 10.3389/fmicb.2018.02757

Löwe, W., and Singer, D. (2008). Quantitative thermographie bei neugeborenen unter verschiedenen arten von wärmestrahlung. 34. Z Geburtshilfe Neonatol. 212:P148.

Marti, H., Blenn, C., and Borel, N. (2015). The contribution of temperature, exposure intensity and visible light to the inhibitory effect of irradiation on acute chlamydial infection. J. Photochem. Photobiol. B Biol. 153, 324-333. doi: 10.1016/j.jphotobiol.2015.10.012

Marti, H., Koschwanez, M., Pesch, T., Blenn, C., and Borel, N. (2014). Waterfiltered infrared a irradiation in combination with visible light inhibits acute chlamydial infection. PLoS One 9:e102239. doi: 10.1371/journal.pone.010 2239

Menezes, S., Coulomb, B., Lebreton, C., and Dubertret, L. (1998). Non-coherent near infrared radiation protects normal human dermal fibroblasts from solar ultraviolet toxicity. J. Invest. Dermatol. 111, 629-633. doi: 10.1046/j.1523-1747. 1998.00338.x

Mercer, J. B., Nielson, S. P., and Hoffmann, G. (2008). Improvement of wound healing by water-filtered infrared-A (wIRA) in patients with chronic venous stasis ulcers of the lower legs including evaluation using infrared thermography. Ger. Med. Sci. 6:Doc11.

Möckel, F., Hoffmann, G., Obermüller, R., Drobnik, W., and Schmitz, G. (2006). Influence of water-filtered infrared-A (wIRA) on reduction of local fat and body weight by physical exercise. Ger. Med. Sci. 4:Doc05.

Notter, M., Piazena, H., and Vaupel, P. (2017). Hypofractionated re-irradiation of large-sized recurrent breast cancer with thermography-controlled, contact-free 
water-filtered infra-red-A hyperthermia: a retrospective study of 73 patients. Int. J. Hyperth. 33, 227-236. doi: 10.1080/02656736.2016.1235731

Otberg, N., Grone, D., Meyer, L., Schanzer, S., Hoffmann, G., Ackermann, H., et al. (2008). Water-filtered infrared-A (wIRA) can act as a penetration enhancer for topically applied substances. Ger. Med. Sci. 6:Doc08.

Passarella, S., and Karu, T. (2014). Absorption of monochromatic and narrow band radiation in the visible and near IR by both mitochondrial and nonmitochondrial photoacceptors results in photobiomodulation This paper is devoted to the memory of Prof. Lorenzo Bolognani who was one of the pioneers in the field of photobiomodulation. J. Photochem. Photobiol. B Biol. 140, 344-358. doi: 10.1016/j.jphotobiol.2014.07.021

Piazena, H., and Kelleher, D. K. (2010). Effects of infrared-A irradiation on skin: discrepancies in published data highlight the need for an exact consideration of physical and photobiological laws and appropriate experimental settings. Photochem. Photobiol. 86, 687-705. doi: 10.1111/j.1751-1097.2010. 00729.x

Rahn, C., Marti, H., Frohns, A., Frohns, F., Blenn, C., Leonard, C. A., et al. (2016). Water-filtered infrared A reduces chlamydial infectivity in vitro without causing ex vivo eye damage in pig and mouse models. J. Photochem. Photobiol. B Biol. 165, 340-350. doi: 10.1016/j.jphotobiol.2016.11.001

Rzeznik, J. (1995). "Die Technik zur loko-regionalen Wärmetherapie mit wassergefilterter Infrarot-A-Strahlung," in Wärmetherapie mit Wassergefilterter Infrarot-A-Strahlung. Grundlagen und Anwendungsmöglichkeiten, eds P. Vaupel and W. Krüger (Stuttgart: Hippokrates Verlag).

Schroeder, P., Lademann, J., Darvin, M. E., Stege, H., Marks, C., Bruhnke, S., et al. (2008). Infrared radiation-induced matrix metalloproteinase in human skin: implications for protection. J. Invest. Dermatol. 128, 2491-2497. doi: 10.1038/ jid.2008.116

Schroeder, P., Pohl, C., Calles, C., Marks, C., Wild, S., and Krutmann, J. (2007). Cellular response to infrared radiation involves retrograde mitochondrial signaling. Free Radic. Biol. Med. 43, 128-135. doi: 10.1016/j.freeradbiomed. 2007.04.002

Singer, D., Schröder, M., and Harms, K. (2000). Vorteile der wassergefilterten gegenüber herkömmlicher Infrarot-Strahlung in der Neonatologie Teil 2 View project Teil 1 View project. Zeitschrift für Geburtshilfe und Neonatalogie 204, 85-92. doi: 10.1055/s-2000-10202

Vaupel, P., Piazena, H., Müller, W., and Notter, M. (2018). Biophysical and photobiological basics of water-filtered infrared-A hyperthermia of superficial tumors biophysical and photobiological basics of water-filtered infrared-A hyperthermia of superficial tumors. Int. J. Hyperth. 35, 2636. doi: $10.1080 / 02656736.2018 .1469169$ org/10.1080/02656736.2018.146 9169

Vaupel, P., Rzeznik, J., and Stofft, E. (1995). Wassergefilterte infrarot-A-strahlung versus konventionelle infrarotstrahlung: temperaturprofile bei lokoregionaler wármetherapie. Phys. Rehabil. Kur. Med. 5, 77-81.

Vogel, M., Bayi, P. F., Ruf, M.-T., Bratschi, M. W., Bolz, M., Um Boock, A., et al. (2016). Local heat application for the treatment of buruli ulcer: results of a phase ii open label single center non comparative clinical trial. Clin. Infect. Dis. 62, 342-350. doi: 10.1093/cid/civ883

Von Felbert, V., Hoffmann, G., Hoff-Lesch, S., Abuzahra, F., Renn, C. N., Braathen, L. R., et al. (2010). Photodynamic therapy of multiple actinic keratoses: reduced pain through use of visible light plus water-filtered infrared A compared with light from light-emitting diodes. Br. J. Dermatol. 163, 607-615. doi: 10.1111/j. 1365-2133.2010.09817.x

Von Felbert, V., Kernland-Lang, K., Hoffmann, G., Wienert, V., Simon, D., and Hunziker, T. (2011). Irradiation with water-filtered infrared A plus visible light improves cutaneous scleroderma lesions in a series of cases. Dermatology 222, 347-357. doi: 10.1159/000329024

von Felbert, V., Schumann, H., Mercer, J. B., Strasser, W., Daeschlein, G., and Hoffmann, G. (2007). Therapy of chronic wounds with water-filtered infraredA (wIRA) therapie chronischer wunden mit wassergefiltertem infrarot A (wIRA). GMS Ger. Med. Sci. 2:Doc52.

Von Felbert, V., Simon, D., Braathen, L. R., Megahed, M., and Hunziker, T. (2007). Behandlung einer linearen sklerodermie mit wassergefilterter infrarotA-strahlung. Der Hautarzt 58, 923-924. doi: 10.1007/s00105-007-1413-y

Conflict of Interest Statement: The authors declare that the research was conducted in the absence of any commercial or financial relationships that could be construed as a potential conflict of interest.

Copyright (c) 2019 Kuratli and Borel. This is an open-access article distributed under the terms of the Creative Commons Attribution License (CC BY). The use, distribution or reproduction in other forums is permitted, provided the original author(s) and the copyright owner(s) are credited and that the original publication in this journal is cited, in accordance with accepted academic practice. No use, distribution or reproduction is permitted which does not comply with these terms. 